\title{
Machine learning classifier to identify clinical and radiological features relevant to disability progression in multiple sclerosis
}

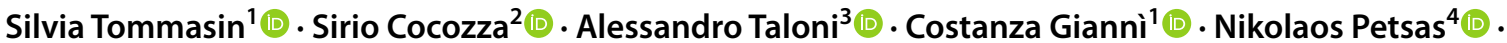

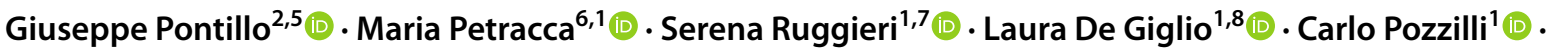 \\ Arturo Brunetti ${ }^{2}$ (i) Patrizia Pantano ${ }^{1,4}$ (i)
}

Received: 16 December 2020 / Revised: 5 May 2021 / Accepted: 5 May 2021 / Published online: 10 May 2021

(c) The Author(s) 2021

\begin{abstract}
Objectives To evaluate the accuracy of a data-driven approach, such as machine learning classification, in predicting disability progression in MS.

Methods We analyzed structural brain images of 163 subjects diagnosed with MS acquired at two different sites. Participants were followed up for 2-6 years, with disability progression defined according to the expanded disability status scale (EDSS) increment at follow-up. T2-weighted lesion load (T2LL), thalamic and cerebellar gray matter (GM) volumes, fractional anisotropy of the normal appearing white matter were calculated at baseline and included in supervised machine learning classifiers. Age, sex, phenotype, EDSS at baseline, therapy and time to follow-up period were also included. Classes were labeled as stable or progressed disability. Participants were randomly chosen from both sites to build a sample including $50 \%$ patients showing disability progression and $50 \%$ patients being stable. One-thousand machine learning classifiers were applied to the resulting sample, and after testing for overfitting, classifier confusion matrix, relative metrics and feature importance were evaluated.

Results At follow-up, $36 \%$ of participants showed disability progression. The classifier with the highest resulting metrics had accuracy of 0.79 , area under the true positive versus false positive rates curve of 0.81 , sensitivity of 0.90 and specificity of 0.71 . T2LL, thalamic volume, disability at baseline and administered therapy were identified as important features in predicting disability progression. Classifiers built on radiological features had higher accuracy than those built on clinical features. Conclusions Disability progression in MS may be predicted via machine learning classifiers, mostly evaluating neuroradiological features.
\end{abstract}

Keywords Multiple sclerosis $\cdot$ Machine learning $\cdot$ Magnetic resonance imaging $\cdot$ Disability progression

\begin{tabular}{llll}
\multicolumn{2}{l}{ Abbreviations } & GM & Gray matter \\
MS & Multiple sclerosis & CIS & Clinically isolated syndrome \\
ML & Machine learning & WM & White matter \\
EDSS & Expanded disability status scale & FA-NAWM & Fractional anisotropy of normal appearing \\
T2LL & T2 lesion load & & white matter
\end{tabular}

Silvia Tommasin

silvia.tommasin@uniroma1.it

1 Department of Human Neurosciences, Sapienza University of Rome, Viale dell'Università, 30, 00185 Rome, Italy

2 Dipartimento di Scienze Biomediche Avanzate, Università degli Studi di Napoli Federico II, Naples, Italy

3 Institute for Complex Systems, Italian National Research Council, Rome, Italy

4 Department of Radiology, IRCCS NEUROMED, Pozzilli, Italy
5 Dipartimento di Ingegneria Elettrica e delle Tecnologie dell'Informazione, Università degli Studi di Napoli Federico II, Naples, Italy

6 Dipartimento di Neuroscienze, Scienze Riproduttive e Odontostomatologiche, Università degli Studi di Napoli Federico II, Naples, Italy

7 Neuroimmunology Unit, IRCSS Fondazione Santa Lucia, Rome, Italy

8 Neurology Unit, Medicine Department, San Filippo Neri Hospital, Rome, Italy 
MD-NAWM Mean diffusivity of normal appearing white matter

AUC Area under the curve clinical and neuroradiological features what ML classifiers are able to identify the most important factors in predicting disability progression in MS.

\section{Materials and methods}

A flowchart showing the methods of this work is proposed in Fig. 1. A detailed step-by-step description of all the procedures follows.

\section{Sample selection}

Study protocols were approved by the ethical committee of Policlinico Umberto I/Sapienza University (Rome, Italy, Site 1) and Ethics Committee for Biomedical Activities "Carlo Romano" of Federico II University (Naples, Italy, Site 2) as appropriate. All subjects provided written informed consent.

We analyzed clinical and MRI data of subjects with MS collected by two centers: the Human Neuroscience Department of Sapienza University (Site 1) and the MS center of the Federico II University (Site 2).

From both sites, clinical evaluations were performed between 2010 and 2018, while all MRI acquisitions were performed between 2010 and 2015. MS patients were included according to the following selection criteria: diagnosis of MS according to the Mc Donald's criteria [20, 21]; age between 18 and 70 years; clinical assessment and MRI examination not more than one month apart; clinical follow-up available after a minimum of 2 years from the MRI examination. On both visits, patients were examined by expert neurologists assessing the clinical status via the Expanded Disability Status Scale (EDSS). At the follow-up examination, disability progression was defined as 1.5 -point increase for patients with a baseline EDSS of 0,1 point for scores from 1.0 to 5.0 , and 0.5 points for scores equal or higher than 5.5 [22].

Finally, at both sites patients underwent a MRI scan with a $3 \mathrm{~T}$ system (Verio, Siemens equipped with a 12-channelhead coil at Site 1; Trio, Siemens, equipped with a 8-channel head coil at Site 2), including tridimensional (3D) T1-weighted (-w); T2-w, either dual echo or FLAIR; diffusion-w images. Detailed information on acquisition parameters are reported in Supplementary Materials.

\section{MRI data analysis}

T2-weighted lesion load (T2LL) was calculated independently by three expert operators, with five to eleven years of experience, who identified the hyperintense WM lesions, using a semi-automatic technique (Jim, Xinapse System, Leicester, UK; http://www.xinapse.com). In the two sites, a lesion mask common to all patients was built including brain 


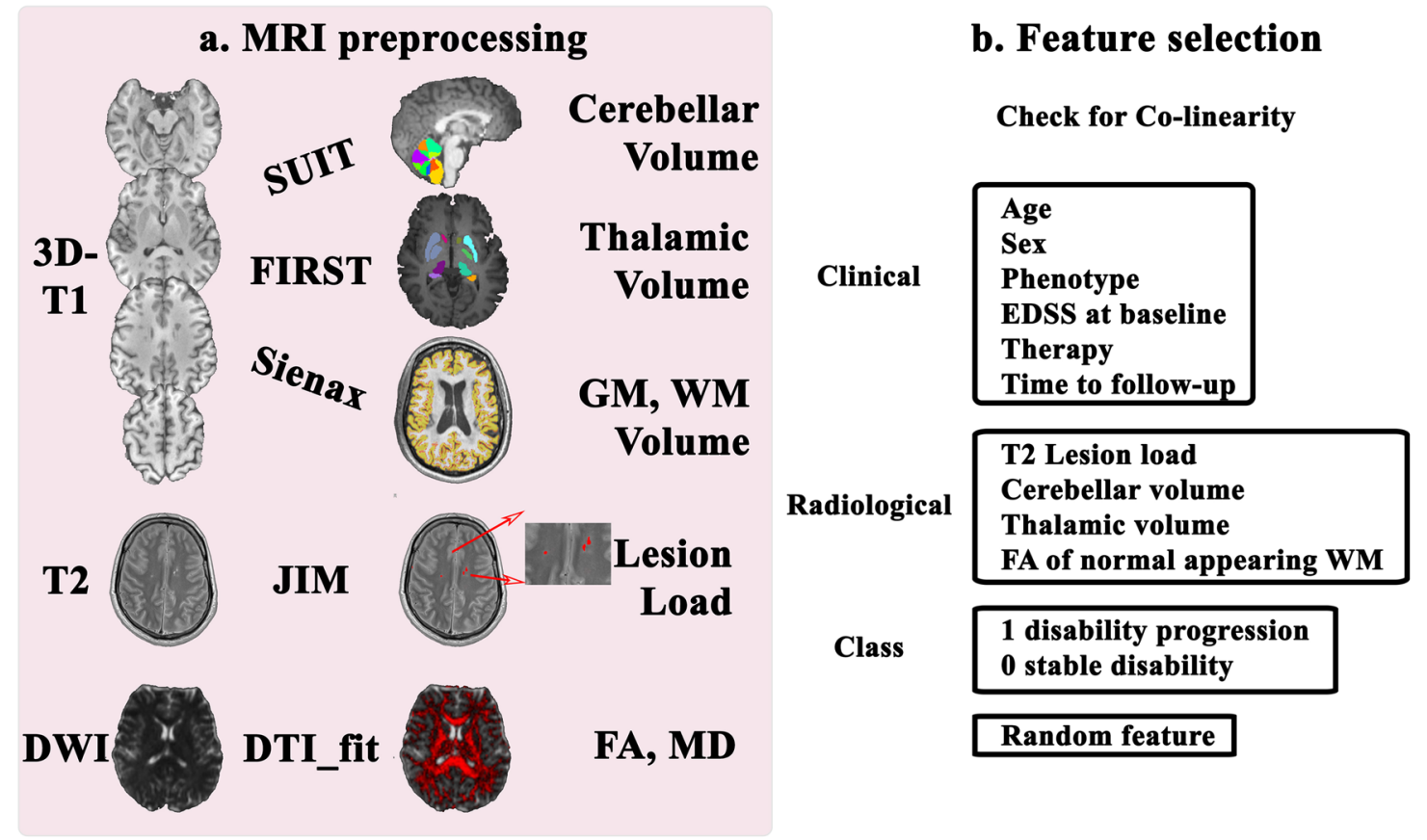

\section{c. ML classifiers}

\begin{abstract}
1000 classifieres built on Clinical +Radiological features 1000 classifiers built on Clinical features 1000 classifiers built on Radiological features
\end{abstract}

\section{Random sample selection}

(50\% class $1,50 \%$ class 0 )

Training set: $80 \%$ of selected sample Validation set: $20 \%$ of selected sample Out-of-Bag test to avoid overfitting Confusion matrix
Fig. 1 Flowchart of the methods. a MRI preprocessing. From 3D-T1weighted images cerebellar volume was calculated via SUIT, thalamic volume using FSL's FIRST and gray matter and white matter volumes via FSL's SIENAX. On the T2-weighted images lesions were identified and segmented using JIM, to calculate the lesion load. By combining lesion and white matter masks, we calculated the normal appearing white matter mask for each subject. From diffusionweighted images both fractional anisotropy and mean diffusivity maps were calculated, and results and were combined with the normal appearing mask previously obtained to extract microstructural metrics of the normal appearing white matter. b Feature selection.

\begin{tabular}{|c|c|c|c|}
\hline & $\begin{array}{l}\text { Predicted } \\
\text { Positive }\end{array}$ & $\begin{array}{l}\text { Predicted } \\
\text { Negative }\end{array}$ & \\
\hline \multirow{2}{*}{$\begin{array}{l}\text { Actual } \\
\text { Positive }\end{array}$} & \multirow{2}{*}{$\begin{array}{l}\text { True } \\
\text { Positive }\end{array}$} & \multirow{2}{*}{$\begin{array}{l}\text { False } \\
\text { Negative }\end{array}$} & $\begin{array}{l}\text { Sensitivity: } \\
\text { True Positive } \\
\text { Positive }\end{array}$ \\
\hline & & & $\begin{array}{l}\text { Specificity: } \\
\text { True Negative } \\
\text { Negative }\end{array}$ \\
\hline \multirow{2}{*}{$\begin{array}{l}\text { Actual } \\
\text { Negative }\end{array}$} & \multirow{2}{*}{$\begin{array}{l}\text { False } \\
\text { Positive }\end{array}$} & \multirow{2}{*}{$\begin{array}{l}\text { True } \\
\text { Negative }\end{array}$} & $\begin{array}{l}\text { Accuracy: } \\
\text { (True Negative+True Positive) } \\
\text { (Positive + Negative) }\end{array}$ \\
\hline & & & $\begin{array}{l}\text { Area under the } \\
\text { Sensitivity vs } \\
\text { Specificity Curve }\end{array}$ \\
\hline
\end{tabular}

Clinical and neuroradiological, were selected together with binary classes (stable patients $=0$, patients with disease progression $=1$ ) and a random feature and used to describe the sample of patients with multiple sclerosis. c Machine learning classifier. After having checked features for co-linearity, a random forest classifier was applied 1000 times feature built on both clinical and radiological features, clinical features alone, radiological features alone. Out-of-Bag test was used to avoid overfitting and performances were evaluated via the confusion matrix of the surviving classifiers. DWI diffusionweighted images, $G M$ gray matter, $W M$ white matter, $F A$ fractional anisotropy, $M D$ mean diffusivity areas presenting lesions in at least the $10 \%$ of the sample [23].

Gray (GM) and white (WM) matter volumes were calculated via SIENAX, implemented in FSL environment (https://fsl.fmrib.ox.ac.uk/fsl/fslwiki/), while thalamic volumes (calculated as the sum of right and left) were obtained via FSL FIRST. Cerebellar lobules, summed to obtain total cerebellar volume, were identified and calculated using the Spatially Unbiased Infratentorial Toolbox (SUIT, http://www.diedrichsenlab.org/imaging/suit.htm), 
implemented in SPM12. Lastly, GM, WM, thalamic and cerebellar volumes were normalized to the scaling factor (obtained in SIENAX) to account for head size.

Diffusion maps were generated using the DTI_fit model, part of the FSL Diffusion Toolbox (http://www.fmrib.ox. ac.uk/fsl/fdt). Combining the results of $\mathrm{FA}$, or MD, for each patient with WM mask and the image of the common lesions, we obtained individual maps of the FA, or MD, for NAWM, that were further averaged to obtain a single value for FA-NAWM and MD-NAWM [23].

\section{Statistical analyses}

Statistical analyses were performed with Matlab R2018b (https://it.mathworks.com/). Group comparison between clinical and radiological features of the two sites was performed using a $\chi$-square to test sex, MS phenotype and disability progression, while a Mann-Whitney non parametric test was used for the remaining variables (age, disease duration, EDSS at baseline, time between visits, T2LL, GM, $\mathrm{WM}$, thalamic and cerebellar volumes, as well as FA- and MD-NAWM values).

To avoid data redundancy and reduce variance in classifier performances, we investigated features' co-linearity of clinical (age, sex, phenotype, disease duration, EDSS at baseline, time between visits and therapy at baseline) and neuroradiological (T2LL, GM, WM, thalamic and cerebellar volumes, FA- and MD-NAWM) features using a partial correlation analysis. Specifically, we performed partial correlation between each pair of features controlling for all the remaining features. For all analyses, a significance threshold was set for $p<0.01$ without multiple comparison correction that allowed us to be conservative in the removal of confounding effects.

\section{ML classifiers}

ML classifiers were performed and analyzed with Python, via the browser version of Jupyter Notebook application (https://jupyter.org/). We used a supervised ML technique and identified two binary classes: patients who were stable (negative output, 0 ) and patients who worsened in disability (positive output, 1) at the follow-up visit. We built a matrix whose rows represented patients and columns represented features. For each patient, features included clinical and neuroradiological data, MR acquisition site and class. A last column consisting of a randomly generated variable was added to the matrix. We applied a random forest algorithm for 1000 times and evaluated the confusion matrices of resulting classifiers.

In each of the 1000 classifiers, we used features calculated on samples from both sites to evaluate classifier performances. Patients from both sites were randomly selected to obtain the same numerosity in both classes $(50 \%$ stable and $50 \%$ with disease progression patients) to avoid bias due to class numerosity. To train the ML classifier we randomly selected $80 \%$ of the sample with the same numerosity of stable and with disease progression patients and left the other $20 \%$ for validation. To avoid overfitting, we calculated each classifier accuracy both on the validation set and in a subsample of the training set and considered in the following analysis only classifiers showing differences in accuracies smaller than 0.02, i.e. Out-of-Bag test. The 0.02 threshold was selected being reasonably low to allow considering the results of the Out-of-Bag test and the training to be consistent, since there is no typical value reported in the literature, at the best of our knowledge.

Classifier performances were evaluated via accuracy, area under the true positive versus false positive rates curve (AUC), sensitivity (true positive rate) and specificity (true negative rate). Within each classifier including both clinical and neuroradiological features, permutation importance was calculated for each feature and for the random variable: only parameters whose importance was higher than the random variable's importance, were considered actually relevant in the classifier.

\section{Results}

\section{Sample selection}

Average and standard deviation of clinical and neuroradiological features for the entire sample built from the two sites, as well as for both Site 1 and Site 2 separately, are reported in Table 1.

In the entire sample, participants were $39.66 \pm 10.23$ years old, range [19.50-70.30], with 104 females and 59 males. Of these 163 subjects, at baseline 122 patients had a relapsing-remitting form of MS, while the remaining 41 patients had a progressive form. Patients showed a disease duration of $9.90 \pm 8.06$ years, range [0.00-37.00], with a time between visits that was $3.93 \pm 0.95$ years, range [2-6]. At the follow-up examination, disease progression was observed in 58 over 163 patients ( $35.6 \%$ of the sample), whose EDSS distributions at baseline and at follow-up were respectively 3.5 [0.0-7.0] and 4.5 [1.5-7.0], while in the remaining 105 patients the EDSS remained stable at 3.0 [0.0-7.5].

Since patients who remained stable at follow-up were about $2 / 3$ of both samples, and we aimed at balancing size of classes, at each of the 1000 performed models we picked all the patients who experienced disability progression from both sites' sample and also randomly picked an equal number of patients who remained stable, reaching a final number of 72 participants from Site 1 and 44 participants from Site 2. 
Table 1 Clinical and neuroradiological features

\begin{tabular}{|c|c|c|c|c|}
\hline & $\begin{array}{l}\text { All subjects (Site } \\
1+\text { Site } 2)\end{array}$ & Subjects at Site 1 & Subjects at Site 2 & Between sites comparison \\
\hline & Average (std) & Average (std) & Average (std) & $z$ - (p-value $)$ \\
\hline Number & 163 & 105 & 58 & - \\
\hline Age [years] & $39.66(10.23)$ & $38.29(9.75)$ & $42.13(10.68)$ & $-2.43(0.02)$ \\
\hline $\operatorname{Sex}(\mathrm{F} / \mathrm{M})$ & $104 / 59$ & $80 / 25$ & $24 / 34$ & $19.61(0.001)^{*}$ \\
\hline Phenotype (RR/P) & $122 / 41$ & $85 / 20$ & $37 / 21$ & $5.84(0.02)^{*}$ \\
\hline Disease duration [years] & $9.90(8.06)$ & $8.27(7.97)$ & $12.87(7.40)$ & $-3.85(0.001)$ \\
\hline EDSS at baseline & $3.0[0.0-7.5]^{* *}$ & $2.0[0.0-7.5]^{* *}$ & $3.5[2.0-7.5]^{* *}$ & $-5.48(0.001)$ \\
\hline Time to follow-up [years] & $3.93(0.95)$ & $4.2(0.93)$ & $3.38(0.72)$ & $6.56(0.001)$ \\
\hline Therapy (1st line, 2nd line, none) & $53,65,45$ & $32,31,42$ & $21,34,3$ & - \\
\hline Disability progression (Yes/No) & $58 / 105$ & $36 / 69$ & $22 / 36$ & $0.22(0.64)^{*}$ \\
\hline $\mathrm{T} 2 \mathrm{LL}[\mathrm{ml}]$ & $9.02(10.31)$ & $6.77(6.94)$ & $13.26(13.62)$ & $-4.47(0.001)$ \\
\hline GM Volume [ml] & $719.69(92.18)$ & $737.38(84.62)$ & $687.662(97.32)$ & $3.48(0.001)$ \\
\hline WM Volume $[\mathrm{ml}]$ & $733.98(96.91)$ & $768.98(84.31)$ & $670.638(85.94)$ & $6.14(0.001)$ \\
\hline Thalamic Volume [ml] & $17.64(3.21)$ & $18.04(3.22)$ & $16.92(3.10)$ & $2.54(0.001)$ \\
\hline Cerebellar Volume [ml] & $113.66(13.88)$ & $110.016(12.92)$ & $120.25(13.22)$ & $-4.28(0.01)$ \\
\hline FA-NAWM & $0.40(0.06)$ & $0.43(0.03)$ & $0.33(0.3)$ & $10.45(0.001)$ \\
\hline MD-NAWM $\times 10^{-3}$ & $0.75(0.05)$ & $0.73(0.07)$ & $0.80(0.04)$ & $-8.68(0.001)$ \\
\hline
\end{tabular}

Clinical and neuroradiological features of Site 1 and Site 2 samples. Mann-Whitney test was used to test significant differences between groups. Significant differences are highlighted in bold font

Std standard deviation, $F$ female; $M$ male; $R R$ relapsing remitting form; $P$ progressive form, EDSS expanded disability status scale, $T 2 L L$ T2 lesion load, GM Gray Matter, WM White Matter, FA-NAWM fractional anisotropy of normal appearing white matter, $M D-N A W M$ mean diffusivity of normal appearing white matter

* $\chi$-square statistics was used

**Median [range]

\section{Feature selection}

Due to differences in demographic and clinical characteristics of participants between the two sites, we performed correlation analysis in the two samples separately. We found a number of features, which were inter-correlated, in both Site 1 and Site 2 samples (Tables 2, 3). After removing intercorrelated features (disease duration, MD-NAWM values, GM and WM volumes), ML classifiers included age, sex, disease phenotype, EDSS score and therapy at baseline, time between visits as clinical features, while T2LL, thalamic and cerebellar volumes and FA-NAWM were included as neuroradiological features.

\section{ML classifiers}

Out of 1000 classifiers built on both clinical and neuroradiological features, 162 classifiers had a difference between the accuracy calculated in the validation set and in the subsample of the training set smaller than 0.02. Accuracy, AUC, sensitivity and specificity on these 162 classifiers are displayed in Fig. 2. The classifier with the highest resulting metrics proved to have an accuracy $=0.79$, an $\mathrm{AUC}=0.81$, with a sensitivity and a specificity of 0.90 and 0.71 , respectively (Table 4).

Then we tested separately classifiers built on neuroradiological features alone (T2LL, FA-NAWM, thalamic and cerebellar volume) and clinical features alone (age, sex, disease phenotype, EDSS score and therapy at baseline, time between visits). For the latter separated analyses, we followed the same procedure implemented to analyze neuroradiological and clinical features together, i.e. balancing the numerosity of class 0 and class 1 , adding a random feature and validating for overfitting via the Out-of-Bag test. We found that classifiers built on neuroradiological features were more accurate and sensitive than those built on clinical features or on mixed clinical-neuroradiological features, showing better accuracy, AUC, sensitivity and specificity values (Table 4; Fig. 3 ).

To have a better depiction of features' role in predicting disability progression, we further investigated the importance of each feature within each classifier built on both clinical and radiological features. T2LL was recognized as important feature in predicting disability progression in all the performed classifiers. EDSS and therapy at baseline were important in the $55 \%$ and $72 \%$ of the classifiers. Thalamic volume and FA-NAWM were important in $39 \%$ and $29 \%$ of 


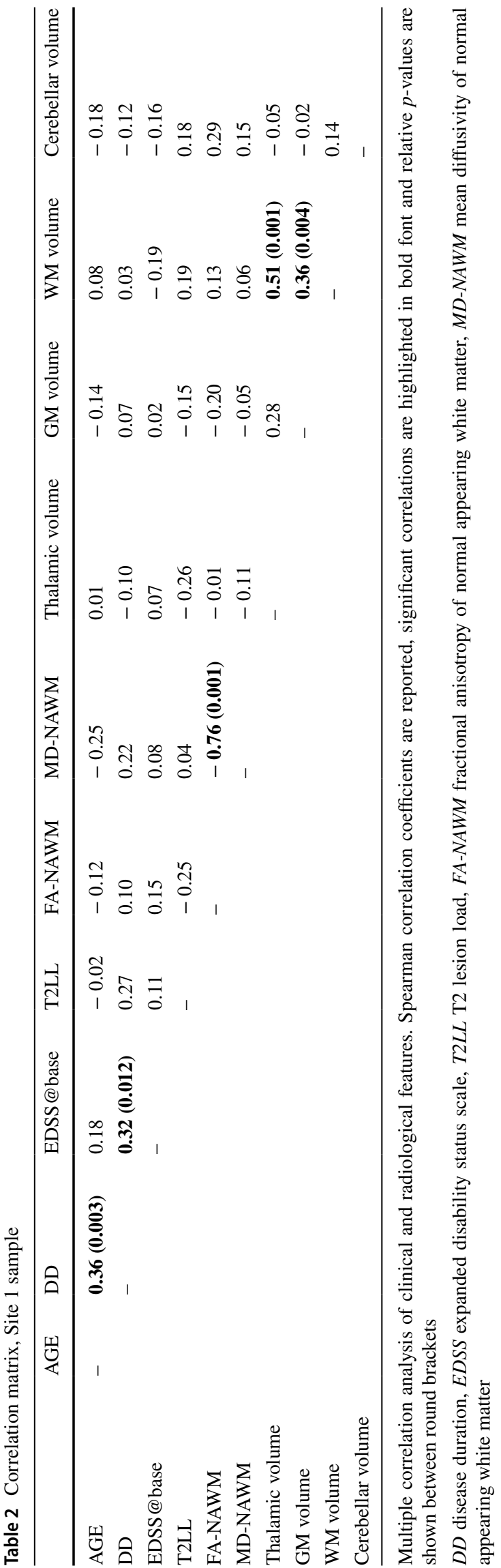

the classifiers respectively. All the other radiological and clinical features were found to be more important than the random feature in a negligible number of classifiers (less than $10 \%$, see Fig. 4).

\section{Discussion}

We showed that MRI measures alone may predict disability progression with the highest accuracy, even in respect to the combination of neuroradiological and clinical features. We are prone to explain this last result as outsourcing from the risk of overfitting. Indeed, the risk of overfitting was higher in the classifiers including both clinical and neuroradiological features due to the numbers of features and subjects and allowed fewer classifiers to survive in respect to the original amount.

Even if we expect that combining both clinical and MRI data may predict clinical outcome at best [24], the role of neuroradiological features seems to be of primary relevance suggesting a more informative independent charge in respect to clinical features alone.

To strengthen the generalizability of the models obtained by the ML classifiers [25], we included in the study features characterizing subjects recruited in two sites, visited by four different clinicians and whose MRI were acquired with two different MR systems. As well, the observed subject samples covered wide ranges of age and disease severity. Therefore, our results may be generalizable and not site-specific, despite recruitment and acquisition protocols from the two sites may have produced differences in feature estimation. Further, we approached our research question with a supervised method, i.e. implementing classification algorithms, and it prevented us to perform less sophisticated methods like principal component analysis.

As Lew et al. [26] showed, ML algorithms may predict 2 -year disability worsening in subjects with progressive disease. Our results extend the previous data, confirming the utility of a ML approach also for subjects in an earlier stage of the disease or with a lower level of disability. This is particularly relevant in this population of MS patients when clinicians have the opportunity to choose among several pharmacological treatments with different mechanism of actions and efficacy outcomes. Indeed, to identify disease features to drive neurologist in their choices is still a matter of debate across literature, given the absence of definitive shared prognostic factors of treatment choose and response [27].

Although we reached $79 \%$ of diagnostic accuracy, our results showed that our model was unable to provide the excellence [28]. Different reasons could explain this finding, ranging from a relatively small sample size to the not-linear relation between structural damage and disability, as it is 


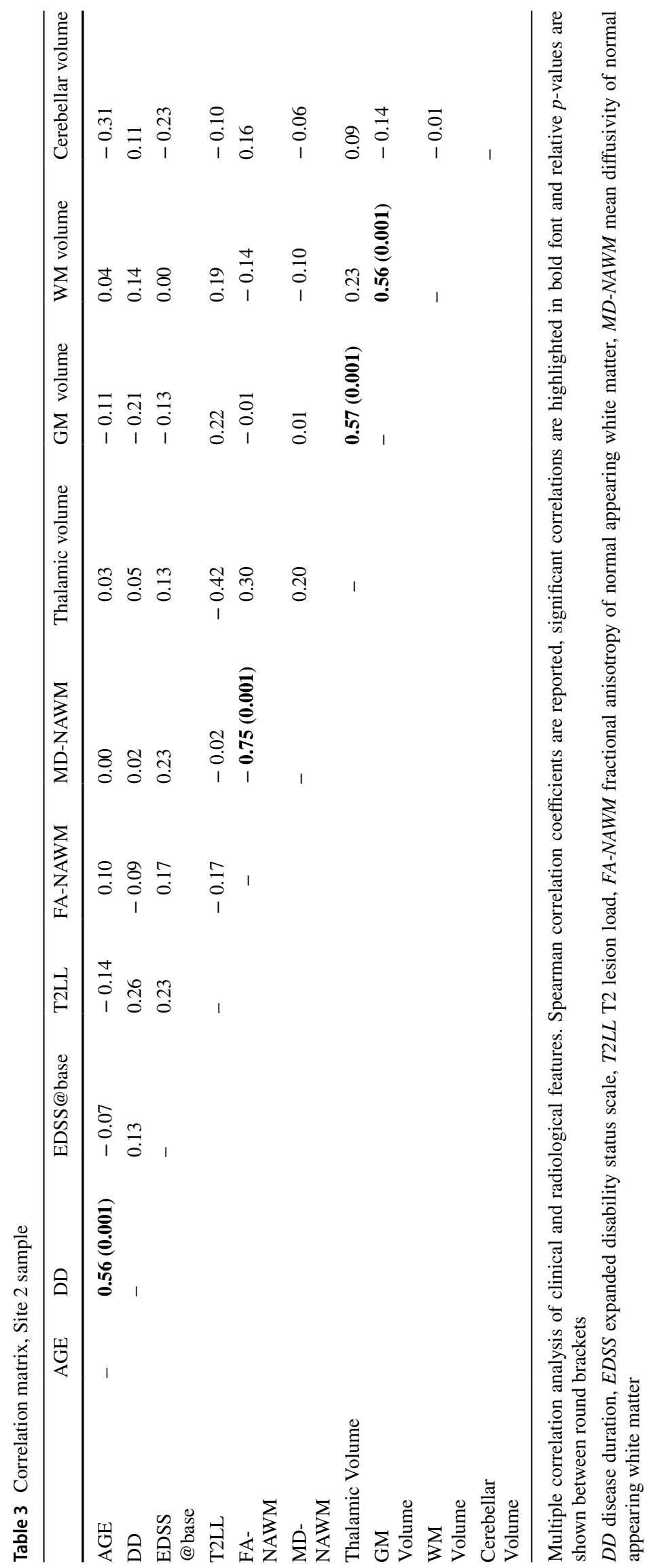



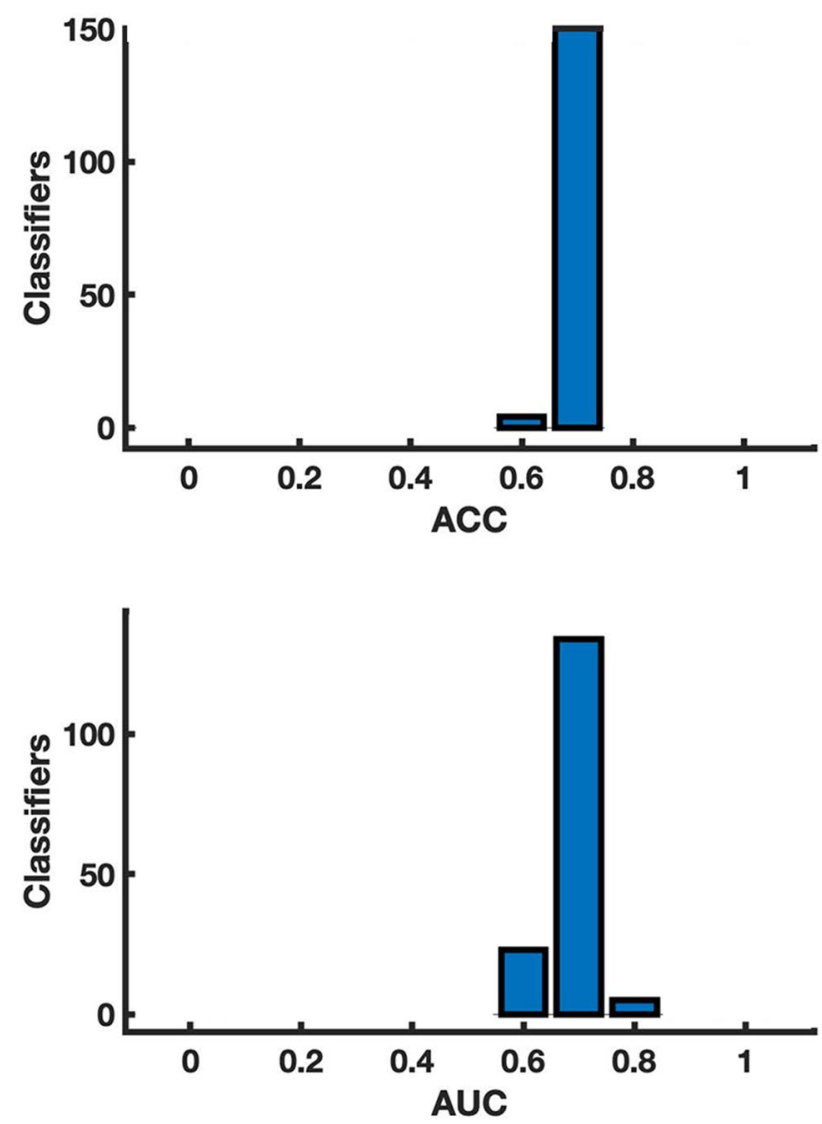

Fig. 2 Metrics of classifier built on clinical and radiological features. Histogram of accuracy (ACC) and area under the true positive versus true negative rate curve (AUC), sensitivity and specificity obtained

Table 4 Metrics

\begin{tabular}{llllll}
\hline & $N$ & Accuracy & AUC & Sensitivity & Specificity \\
\hline ALL & 162 & 0.79 & 0.81 & 0.90 & 0.71 \\
Radiological & 329 & 0.92 & 0.92 & 0.92 & 0.91 \\
Clinical & 128 & 0.71 & 0.72 & 0.69 & 0.75 \\
\hline
\end{tabular}

Accuracy, area under the true positive versus true negative curve (AUC), sensitivity and specificity of the best performing machine learning classifier built on all clinical and radiological features, and either on clinical or radiological features. $N$ represents the number of classifiers surviving the Out-of-Bag test

quantified by the EDSS [29]. Nevertheless, it has to be considered that a sample size of 116 subjects may be enough to model disability progression from clinical and radiological features, as previously found [23, 30, 31]. Furthermore, our analysis included only parameters of structural brain damage, while the association between clinical findings and radiological extent of involvement is generally poor and known, given the clinico-radiological paradox in MS [32].

With regards to MRI data, our results indirectly confirm the importance of T2LL in predicting disease worsening,
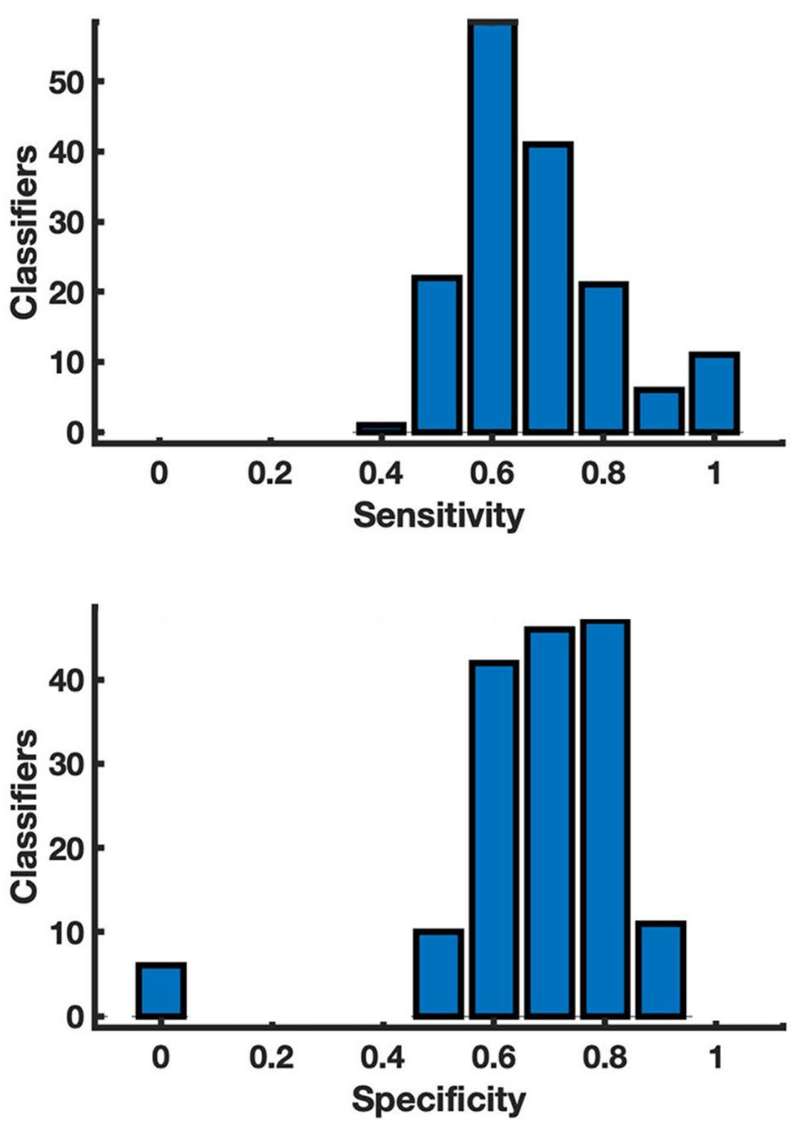

from the 150 classifiers, surviving the Out-of-Bag test, performed on the sample features. On the $y$-axis number of classifiers is displayed

a variable known to influence both the disease course [24] and its progression $[14,33]$. In particular, following the first episode of the disease, T2LL is helpful to identify those patients at risk of developing an aggressive form of MS [34]. This underlines how our result can help physicians in selecting those patients that can benefit from an early and more efficacy therapy. A recent study by Bakshi and colleagues found that baseline brain parenchymal fraction, but not T2LL, may be a good predictor of 5-year disability worsening [35]. A possible explanation to this discrepancy could be researched in the different clinical endpoint. In particular, authors considered longitudinal difference in the EDSS score as a disability marker, while we considered disability progression as defined in Rìo et al. [22]. Further, our results confirm the prognostic role of thalamus, whose measurement is reliable and comparable among centers, in MS [36]. Indeed, thalamic involvement in MS has been widely investigated using different approaches, with thalamic atrophy and function associated with poorer motor and cognitive performances [37, 38]. A recent and extensive work in 1417 subjects with MS identified key regions with early atrophy such thalamus consistently across MS phenotypes, exploring 


\section{Radiological Features}
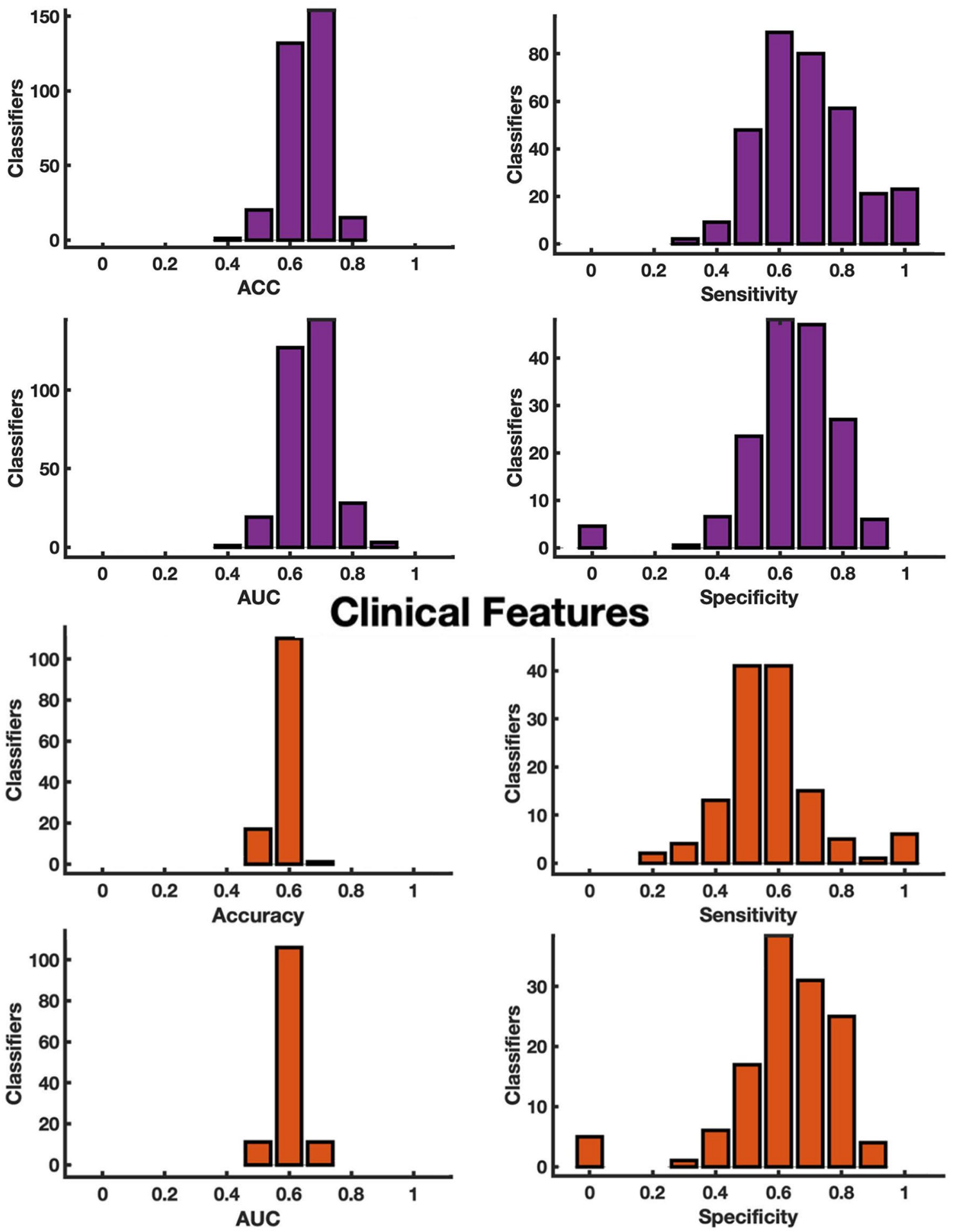

Fig. 3 Metrics of classifier built on radiological/clinical features. Histograms of accuracy (ACC) and area under the true positive versus true negative rate curve (AUC), sensitivity and specificity, surviving

the Out-of-Bag test obtained from the 309 classifiers built on neuroradiological features (top) and the 128 classifiers built on clinical features (bottom). On the y-axis number of classifiers is displayed 


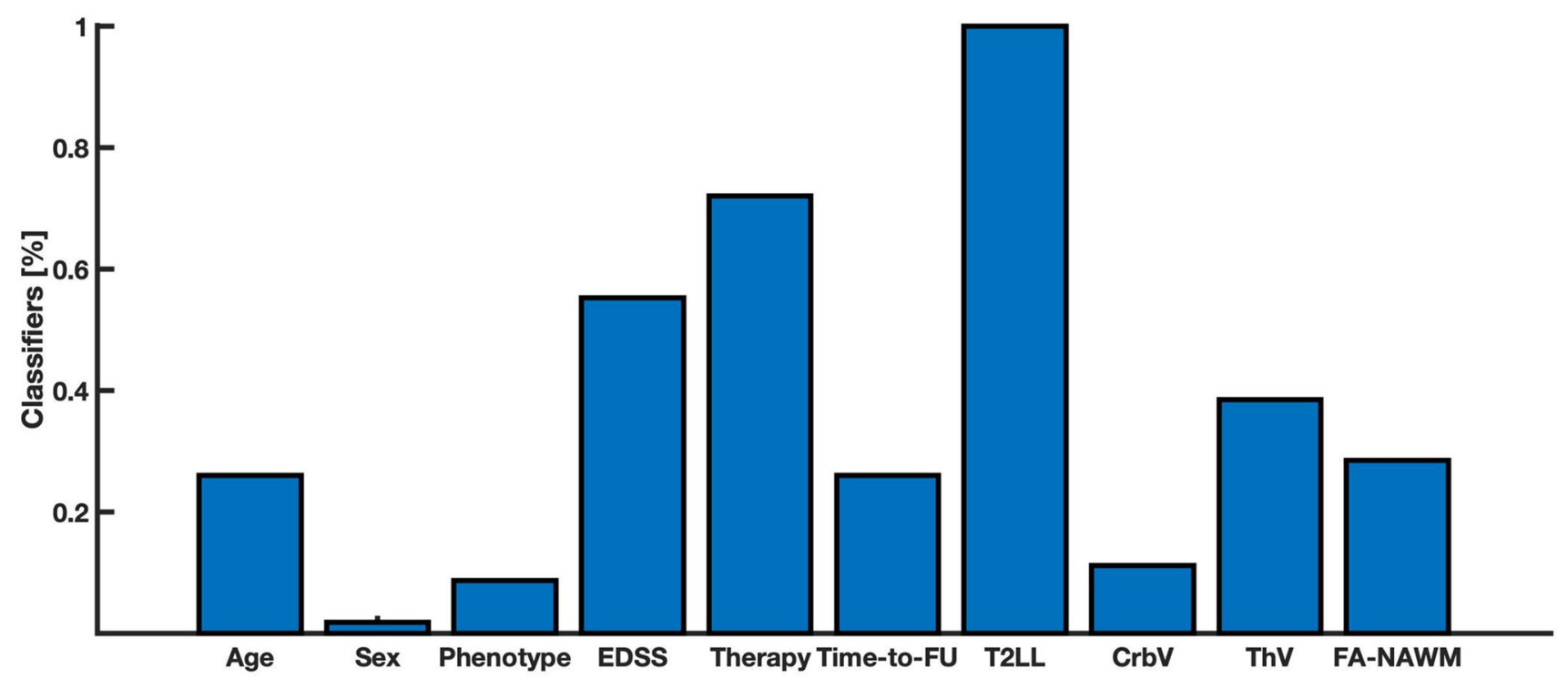

Fig. 4 Important features frequency. Figure showing, for each feature, the percentage of time (i.e. classifiers) more important than the random feature in predicting disability progression in our MS group. EDSSbase expanded disability status scale at baseline, TimeFU time

the sequence of GM atrophy and revealing the potentiality of therapeutic strategy adjustment based on the staging of patients [10,39]. Finally, FA-NAWM values also proved to be a significant contributor to clinical progression in MS, in line with the established knowledge about the association between axonal loss and clinical deficit in MS [40], as well as that aberrant diffusivity measures in the NAWM may provide information in the relapsing remitting form of MS [41].

Lastly, in our analysis, both disability and therapy at baseline emerged as clinical features relevant to disability progression. In particular, therapy response is individual and depends on many factors, including age, phenotype and disability at treatment start [42], and is known to influence both disease course [43] and disability accumulation [44].

\section{Limitations}

As mentioned before, a limitation of our study relies in the relatively small sample size, which might have affected the performance of ML classifiers in the prediction of MS disability, limiting their diagnostic accuracy. The small sample size also prevented us from using an independent test set to report final results [25]. Further, we used EDSS as index of disability. Even if depending mainly on walking ability, EDSS is widely used as disability measure [45]. More in general, the major limitation of this study has to be researched in its retrospective nature. For this reason, future prospective studies, properly designed to evaluate with additional techniques (e.g. fMRI, MTR, qMRI) other aspects between first visit and follow-up, $C r b V$ cerebellar gray matter volume, GMV gray matter volume, FA-NAWM fractional anisotropy of normal appearing white matter

of brain involvement, as well as other CNS structures (e.g. spinal cord) are strongly warranted, to verify our model and possibly increase its diagnostic accuracy.

\section{Conclusion}

ML classifiers built on clinical and neuroradiological features may predict disability progression in subjects with MS at individual level with accuracy almost reaching a value of $80 \%$. Among the MRI variables, T2LL and thalamic volume were the most important features in the prediction of disability progression, while disability and therapy at baseline were the most relevant features among the clinical variables. The implementation of classifiers based on neuroradiological and clinical features may aid clinicians to predict if a single subject may be prone to disability progression and consequently to tailor the treatment.

Supplementary Information The online version contains supplementary material available at https://doi.org/10.1007/s00415-021-10605-7.

Funding Open access funding provided by Università degli Studi di Roma La Sapienza within the CRUI-CARE Agreement. This study has received funding by Fondazione Italiana Sclerosi Multipla (FISM), Grant FISM/2018/S/3.

\section{Declarations}

Conflicts of interest ST, AT, CG, GP, AB have nothing to disclose. SC: received speaker fees from Sanofi and Amicus Therapeutics. NP: 
received speaker fees from Biogen and mission support from Genzyme and Novartis. MP: received travel funding from Novartis. SR: received speaking honoraria from Teva, Merck Serono, and Biogen, a travel grant from Biogen and Merck Serono, and a fee as an advisory board consultant from Merck Serono and Novartis. LDG: received fees as invited speaker or travel expenses for attending meeting from Biogen, Merck Serono and Novartis. CP: received consulting and lecture fees from Sanofi-Aventis, Biogen Idec, Bayer Schering, Merck Serono, and Novartis, research funding from Novartis, Sanofi-Aventis, Merck Serono, and Bayer Schering. PP: received founding for travel from Novartis, Genzyme and Bracco and speaker honoraria from Biogen.

Ethics approval Study protocols were approved by the ethical committee of PoliclinicoUmberto I/Sapienza University (Rome, Italy, Site 1) and Ethics Committee for Biomedical Activities "Carlo Romano" of Federico II University (Naples, Italy, Site 2) as appropriate.

Informed consent All subjects provided written informed consent.

Open Access This article is licensed under a Creative Commons Attribution 4.0 International License, which permits use, sharing, adaptation, distribution and reproduction in any medium or format, as long as you give appropriate credit to the original author(s) and the source, provide a link to the Creative Commons licence, and indicate if changes were made. The images or other third party material in this article are included in the article's Creative Commons licence, unless indicated otherwise in a credit line to the material. If material is not included in the article's Creative Commons licence and your intended use is not permitted by statutory regulation or exceeds the permitted use, you will need to obtain permission directly from the copyright holder. To view a copy of this licence, visit http://creativecommons.org/licenses/by/4.0/.

\section{References}

1. Confavreux C, Vukusic S (2014) The clinical course of multiple sclerosis. Handb Clin Neurol 122:343-369. https://doi.org/10. 1016/B978-0-444-52001-2.00014-5

2. Ciccarelli O, Barkhof F, Bodini B et al (2014) Pathogenesis of multiple sclerosis: insights from molecular and metabolic imaging. Lancet Neurol 13:807-822. https://doi.org/10.1016/S14744422(14)70101-2

3. Amato MP, Fonderico M, Portaccio E et al (2020) Disease-modifying drugs can reduce disability progression in relapsing multiple sclerosis. Brain. https://doi.org/10.1093/brain/awaa251

4. Tommasin S, Giannì C, De Giglio L, Pantano P (2017) Neuroimaging techniques to assess inflammation in multiple sclerosis. Neuroscience. https://doi.org/10.1016/j.neuroscience.2017.07.055

5. Tintore M, Rovira À, Río J et al (2015) Defining high, medium and low impact prognostic factors for developing multiple sclerosis. Brain 138:1863-1874. https://doi.org/10.1093/brain/awv105

6. Pontillo G, Cocozza S, Di Stasi M et al (2020) 2D linear measures of ventricular enlargement may be relevant markers of brain atrophy and long-term disability progression in multiple sclerosis. Eur Radiol. https://doi.org/10.1007/s00330-020-06738-4

7. Radue E-W, Barkhof F, Kappos L et al (2015) Correlation between brain volume loss and clinical and MRI outcomes in multiple sclerosis. Neurology 84:784-793. https://doi.org/10.1212/WNL. 0000000000001281

8. Louapre C, Bodini B, Lubetzki C et al (2017) Imaging markers of multiple sclerosis prognosis. Curr Opin Neurol 30:231-236. https://doi.org/10.1097/WCO.0000000000000456

9. Filippi M, Brück W, Chard D et al (2019) Association between pathological and MRI findings in multiple sclerosis. Lancet
Neurol 18:198-210. https://doi.org/10.1016/S1474-4422(18) 30451-4

10. Eshaghi A, Prados F, Brownlee WJ et al (2018) Deep gray matter volume loss drives disability worsening in multiple sclerosis. Ann Neurol 83:210-222. https://doi.org/10.1002/ana.25145

11. Cocozza S, Petracca M, Mormina E et al (2017) Cerebellar lobule atrophy and disability in progressive MS. J Neurol Neurosurg Psychiatry 88:1065-1072. https://doi.org/10.1136/jnnp-2017-316448

12. D'Ambrosio A, Pagani E, Riccitelli GC et al (2017) Cerebellar contribution to motor and cognitive performance in multiple sclerosis: an MRI sub-regional volumetric analysis. Mult Scler 23:1194-1203. https://doi.org/10.1177/1352458516674567

13. Patti F, De Stefano M, Lavorgna L et al (2015) Lesion load may predict long-term cognitive dysfunction in multiple sclerosis patients. PLoS ONE 10:e0120754. https://doi.org/10.1371/journ al.pone.0120754

14. Calabrese M, Poretto V, Favaretto A et al (2012) Cortical lesion load associates with progression of disability in multiple sclerosis. Brain 135:2952-2961. https://doi.org/10.1093/brain/aws246

15. Wilkins A (2017) Cerebellar dysfunction in multiple sclerosis. Front Neurol. https://doi.org/10.3389/fneur.2017.00312

16. Rocca MA, Mesaros S, Pagani E et al (2010) Thalamic damage and long-term progression of disability in multiple sclerosis. Radiology 257:463-469. https://doi.org/10.1148/radiol.10100326

17. Datta G, Colasanti A, Rabiner EA et al (2017) Neuroinflammation and its relationship to changes in brain volume and white matter lesions in multiple sclerosis. Brain 140:2927-2938. https://doi.org/10.1093/brain/awx228

18. Wottschel V, Alexander DC, Kwok PP et al (2015) Predicting outcome in clinically isolated syndrome using machine learning. Neuroimage Clin 7:281-287. https://doi.org/10.1016/j.nicl. 2014.11.021

19. Zurita M, Montalba C, Labbé T et al (2018) Characterization of relapsing-remitting multiple sclerosis patients using support vector machine classifications of functional and diffusion MRI data. Neuroimage Clin 20:724-730. https://doi.org/10.1016/j. nicl.2018.09.002

20. Polman CH, Reingold SC, Banwell B et al (2011) Diagnostic criteria for multiple sclerosis: 2010 revisions to the McDonald criteria. Ann Neurol 69:292-302. https://doi.org/10.1002/ana. 22366

21. Thompson AJ, Banwell BL, Barkhof F et al (2018) Diagnosis of multiple sclerosis: 2017 revisions of the McDonald criteria. Lancet Neurol 17:162-173. https://doi.org/10.1016/S1474-4422(17) 30470-2

22. Río J, Nos C, Tintoré $M$ et al (2006) Defining the response to interferon-beta in relapsing-remitting multiple sclerosis patients. Ann Neurol 59:344-352. https://doi.org/10.1002/ana.20740

23. Raz E, Cercignani M, Sbardella E et al (2009) Clinically isolated syndrome suggestive of multiple sclerosis: voxelwise regional investigation of White and Gray matter. Radiology 254:227-234. https://doi.org/10.1148/radiol.2541090817

24. Zhao Y, Healy BC, Rotstein D et al (2017) Exploration of machine learning techniques in predicting multiple sclerosis disease course. PLoS ONE 12:e0174866. https://doi.org/10.1371/journal.pone. 0174866

25. Bluemke DA, Moy L, Bredella MA et al (2020) Assessing radiology research on artificial intelligence: a brief guide for authors, reviewers, and readers - from the radiology editorial board. Radiology 294:487-489. https://doi.org/10.1148/radiol.2019192515

26. Law MT, Traboulsee AL, Li DK et al (2019) Machine learning in secondary progressive multiple sclerosis: an improved predictive model for short-term disability progression. Mult Scler J Exp Transl Clin 5:2055217319885983. https://doi.org/10.1177/20552 17319885983 
27. Gasperini C, Prosperini L, Tintoré M et al (2019) Unraveling treatment response in multiple sclerosis: a clinical and MRI challenge. Neurology 92:180-192. https://doi.org/10.1212/WNL.00000 00000006810

28. Šimundić A-M (2009) Measures of diagnostic accuracy: basic definitions. EJIFCC 19:203-211

29. Schoonheim MM, Geurts JJG, Barkhof F (2010) The limits of functional reorganization in multiple sclerosis. Neurology 74:1246-1247. https://doi.org/10.1212/WNL.0b013e3181db9957

30. Calabrese M, Mattisi I, Rinaldi F et al (2010) Magnetic resonance evidence of cerebellar cortical pathology in multiple sclerosis. J Neurol Neurosurg Psychiatry 81:401-404. https://doi.org/10. 1136/jnnp.2009.177733

31. Davie CA, Barker GJ, Webb S et al (1995) Persistent functional deficit in multiple sclerosis and autosomal dominant cerebellar ataxia is associated with axon loss. Brain 118(Pt 6):1583-1592. https://doi.org/10.1093/brain/118.6.1583

32. Barkhof $F$ (2002) The clinico-radiological paradox in multiple sclerosis revisited. Curr Opin Neurol 15:239-245

33. Elliott C, Belachew S, Wolinsky JS et al (2019) Chronic white matter lesion activity predicts clinical progression in primary progressive multiple sclerosis. Brain 142:2787-2799. https://doi.org/ 10.1093/brain/awz212

34. Tintore M, Arrambide G, Otero-Romero S et al (2019) The longterm outcomes of CIS patients in the Barcelona inception cohort: Looking back to recognize aggressive MS. Mult Scler. https://doi. org/10.1177/1352458519877810

35. Bakshi R, Healy BC, Dupuy SL et al (2020) Brain MRI predicts worsening multiple sclerosis disability over 5 years in the SUMMIT study. J Neuroimaging. https://doi.org/10.1111/jon.12688

36. Dwyer M, Brior D, Lyman C et al (2020) Artificial intelligence-based thalamic volumetry is fast, reliable, and generalizable to large, heterogeneous datasets using only clinical quality T2-FLAIR MRI (4846). Neurology 94:4846
37. Azevedo CJ, Cen SY, Khadka S et al (2018) Thalamic atrophy in multiple sclerosis: a magnetic resonance imaging marker of neurodegeneration throughout disease. Ann Neurol 83:223-234. https://doi.org/10.1002/ana.25150

38. Tona F, Petsas N, Sbardella E et al (2014) Multiple sclerosis: altered thalamic resting-state functional connectivity and its effect on cognitive function. Radiology 271:814-821. https://doi.org/10. 1148/radiol.14131688

39. Stankoff B, Louapre C (2018) Can we use regional grey matter atrophy sequence to stage neurodegeneration in multiple sclerosis? Brain 141:1580-1583. https://doi.org/10.1093/brain/awy114

40. Haines JD, Inglese M, Casaccia P (2011) axonal damage in multiple sclerosis. Mt Sinai J Med 78:231-243. https://doi.org/10.1002/ msj.20246

41. Kolasa M, Hakulinen U, Brander A et al (2019) Diffusion tensor imaging and disability progression in multiple sclerosis: a 4-year follow-up study. Brain Behav 9:e01194. https://doi.org/10.1002/ brb3.1194

42. Kalincik T, Manouchehrinia A, Sobisek L et al (2017) Towards personalized therapy for multiple sclerosis: prediction of individual treatment response. Brain 140:2426-2443. https://doi.org/ 10.1093/brain/awx185

43. Cree BAC, Mares J, Hartung H-P (2019) Current therapeutic landscape in multiple sclerosis: an evolving treatment paradigm. Curr Opin Neurol 32:365-377. https://doi.org/10.1097/WCO.00000 00000000700

44. Hart FM, Bainbridge $\mathrm{J}$ (2016) Current and emerging treatment of multiple sclerosis. Am J Manag Care 22:s 159-170

45. Cohen JA, Reingold SC, Polman CH et al (2012) Disability outcome measures in multiple sclerosis clinical trials: current status and future prospects. Lancet Neurol 11:467-476. https://doi.org/ $10.1016 /$ S1474-4422(12)70059-5 